\title{
Effects of magnetic field on the plasma evolution in relativistic heavy-ion collisions
}

\author{
Arpan Das ${ }^{1,2} *$, Shreyansh S. Dave ${ }^{1,2} \dagger$, P.S. Saumia ${ }^{\ddagger} \ddagger$, and Ajit M. Srivastava ${ }^{1,2} \S$ \\ 1 Institute of Physics, Bhubaneswar 751005, India \\ ${ }^{2}$ Homi Bhabha National Institute, Training School Complex, Anushakti Nagar, Mumbai 400085, India \\ 3 Bogoliubov Laboratory of Theoretical Physics, JINR, 141980, Dubna, Russia
}

\begin{abstract}
Very strong magnetic fields can arise in non-central heavy-ion collisions at ultrarelativistic energies, which may not decay quickly in a conducting plasma. We carry out relativistic magnetohydrodynamics (RMHD) simulations to study the effects of this magnetic field on the evolution of the plasma and on resulting flow fluctuations in the ideal RMHD limit. Our results show that magnetic field leads to enhancement in elliptic flow for small impact parameters while it suppresses it for large impact parameters (which may provide a signal for initial stage magnetic field). Interestingly, we find that magnetic field in localized regions can temporarily increase in time as evolving plasma energy density fluctuations lead to reorganization of magnetic flux. This can have important effects on chiral magnetic effect. Magnetic field has non-trivial effects on the power spectrum of flow fluctuations. For very strong magnetic field case one sees a pattern of even-odd difference in the power spectrum of flow coefficients arising from reflection symmetry about the magnetic field direction if initial state fluctuations are not dominant. We discuss the situation of nontrivial magnetic field configurations arising from collision of deformed nuclei and show that it can lead to anomalous elliptic flow. Special (crossed body-body) configurations of deformed nuclei collision can lead to presence of quadrupolar magnetic field which can have very important effects on the rapidity dependence of transverse expansion (similar to beam focusing from quadrupole fields in accelerators).

PACS numbers: PACS numbers: 11.27.+d, 98.80.Cq, 25.75.-q
\end{abstract}

\section{INTRODUCTION}

Extensive efforts have focused on the discovery of the quark-gluon plasma (QGP) phase of QCD in relativistic heavy-ion collision experiments (RHICE). There is mounting evidence that QGP phase is created in these experiments. It is no more possible to explain the wealth of experimental data at RHIC and LHC without assuming a transient phase of QGP. While it is certainly desirable to have smoking gun signal for QGP, it is also an appropriate stage for the exploration of the rich spectrum of physics unfolded by the (most likely) presence of this transient stage of QGP in relativistic heavy-ion collisions. Search for exciting possibilities like the critical point in the QCD phase diagram, possible exotic high baryon density phases (in upcoming facilities FAIR and NICA) are some of these directions.

An entire new line of explorations has been initiated in recent years by the very exciting possibility that in relativistic heavy-ion collision experiments extremely high magnetic fields are expected to arise, especially in noncentral collisions. During earliest stages, magnetic field in the plasma can be of order $10^{15}$ Tesla (few $m_{\pi}^{2}$ ), which is several orders of magnitude larger than the magnetic field even in magnetars. Such a strong magnetic field in QGP will lead to important effects. Much of the discussion in literature has focused on the exciting possibility

\footnotetext{
*email: arpan@iopb.res.in

†email: shreyansh@iopb.res.in

‡email: saumia@theor.jinr.ru

$\S$ email: ajit@iopb.res.in
}

of observing $\mathrm{CP}$ violation effects 1. Relevant effects are generally known as chiral magnetic effect and more recently discussed chiral vortical effect. Along with such effects, there are many other important consequences of the magnetic field for QGP evolution. Some of us had earlier utilized the fact that an important effect of the presence of magnetic fields in the plasma will be to lead to variations in velocities of different types of waves in the plasma [3. In particular the group velocity varies with the angle between the wave vector and the direction of the magnetic field. Its obvious effect will be to qualitatively modify the development of anisotropic flow. In ref. 3, it was argued that the flow coefficients can be significantly affected by these effects, in particular, the presence of magnetic field can lead to enhancement in the elliptic flow coefficient $v_{2}$ by almost $30 \%$. As pointed out in ref. 3], it raises the interesting possibility of whether a larger value of $\eta / s$ can be accommodated by RHIC data when these effects are incorporated using full magnetohydrodynamical simulations. This possibility can be viewed as either leading to the QGP $\eta / s$ being higher than the AdS/CFT bound, or in the context of results in ref. 4. which suggested crossing the AdS/CFT bound, to restore the bound when proper effects of magnetic field are incorporated. The issue of magnetic field dependence of elliptic flow was discussed by Tuchin [5] (including viscous effects as well) with results in agreement with [3].

The arguments in ref. 3 utilized directional dependence of sound velocity in the presence of magnetic field and modeled its effect on development of elliptic flow. Those results were not based on any magnetohydrodynamical simulation. In this paper, we have carried out detailed relativistic magnetohydrodynamics simulations. 
We indeed confirm the results in $[3,5$, that elliptic flow can increase in the presence of magnetic field. However, our results show that the dependence of $v_{2}$ on magnetic field is much more complex than assumed in these earlier works, with several factors at play. In certain situations (e.g. for small impact parameters) the magnetic field enhances the elliptic flow, while in a different situation (large impact parameter), magnetic field suppresses the elliptic flow. These underlying factors are important to understand (especially in view of other recent relativistic magnetohydrodynamics simulations $[6$ where it was found that magnetic field has no effect on elliptic flow, in contrast to the results in [3, 5]). Along with the effect on elliptic flow, we will demonstrate several other important effects of magnetic field showing how flow evolution is qualitatively affected. These effects are important as they show that an understanding of flow pattern is not complete without including effects of magnetic field in the early stages of plasma evolution.

Another important reason to focus on different qualitative effects of magnetic field on flow evolution is that it can provide signal for the presence of strong magnetic field during early stages of the plasma evolution. It must be emphasized that although for the earliest stage of collision, magnetic field can be calculated with reasonably accurate approximations, its evolution even in immediately successive stages is poorly understood. All the important effects of the magnetic field, such as chiral magnetic effect as well as various effects on flow pattern as we have discussed here (and in [3, 5]) require that reasonably strong magnetic field survives for at least several fm proper time. Earlier it was thought that magnetic field rapidly decays after the collision. It is strong for a very short time, essentially the passing time of the Lorentz contracted nuclei ( $\sim 0.2 \mathrm{fm}$ for RHIC energies). Subsequently it rapidly decays [2, 7]. In such a situation the effect of magnetic field on flow evolution as well effects such as chiral magnetic effect will be strongly suppressed as time scale for the development of flow and for charge separation (for latter effects) is several fm. Similar situation is expected at higher energies, e.g. at LHC.

It was later pointed out by Tuchin $[8$ that magnetic field does not decay very rapidly due to induced currents arising from rapidly decreasing external magnetic field. In fact, the magnetic field satisfies a diffusion equation with the diffusion constant equal to $1 /(\sigma \mu)$ where $\mu$ is the magnetic permeability and $\sigma$ is the electrical conductivity [9, 10. With $\mu \sim 1$ and $\sigma \simeq 0.04 T\left(=0.04 \mathrm{fm}^{-1}\right.$ for T $\simeq 200 \mathrm{MeV}$ ) from refs. 11], one finds that the time scale $\tau$ over which the magnetic field remains reasonably strong [8] over length scale $L$ is, $\tau \simeq L^{2} \sigma / 4$. For $L=6-10 \mathrm{fm}$, we get $\tau$ less than $1 \mathrm{fm}$. Indeed, one sees that magnetic field decreases fast initially, though at later times matter effects become more important slowing down decrease of magnetic field significantly [12]. For higher temperatures $\sigma$ will be larger increasing the value of $\tau$. $\sigma$ is also expected to increase due to the effects of magnetic field in the plasma [13, further increasing the value of $\tau$.
Even if one takes this optimistic picture that due to non-zero conductivity of QGP, magnetic field doe not decay extremely rapidly, and may survive for significant time scales, the self-consistency of this picture can be questioned due to uncertainties of the initial nonequilibrium stages of the parton system. Initially there is no plasma, so no conductivity. If the parton system is assumed to have the QGP conductivity during its formation stages, question arises as to how magnetic field can penetrate the conducting plasma. For simplicity consider the plasma (say during early stage for less than $1 \mathrm{fm}$ lab time) to be a thick disk of nuclear diameter and thickness of 1-2 fm. If the plasma was static then one could just consider the penetration depth $\delta \sim(\mu \sigma \omega)^{-1 / 2}$ where $\omega$ is the angular frequency of electromagnetic wave. Initial magnetic field, being a narrow pulse of time duration $t \simeq 0.2 \mathrm{fm}$ (typically the width of Lorentz contracted Nuclei, for RHIC energies), can be taken to have $\omega \simeq 30$ $\mathrm{fm}^{-1}$. This gives the penetration depth of order $1 \mathrm{fm}$ (note it was mistakenly written as $3 \mathrm{fm}$ in ref.[3]). In such a situation, though magnetic field cannot penetrate from the perimeter of the disc (nuclear radius being about 6-7 $\mathrm{fm}$ ), it may be able to penetrate significantly in the interior from the longitudinal direction (from both sides), with disk thickness being only about $1 \mathrm{fm}$. In such a situation, the picture of magnetic field diffusing through the entire region of the plasma with typical length scale of several $\mathrm{fm}$, and lasting with a value close to the high initial peak values for time scales of several fm, may be self consistent.

However, the plasma is not static in the longitudinal direction. Far from it, the plasma is relativistically expanding in the longitudinal direction. The above argument of penetration depth cannot be applied to a conducting plasma which is relativistically expanding. The conclusion being that if the plasma is taken to be conducting from the very beginning, we do not know how much fraction of the original magnetic field penetrates the plasma. Only that fraction can then be assumed to follow the diffusion equation as in ref. 8 , and survive for few fm time scale. A proper treatment of the problem will require treatment of the early parton system as a nonequilibrium system, whose response to ambient magnetic field then needs to be estimated. As the plasma equilibrates, it will develop conductivity as appropriate for the QGP phase, and one needs to determine how much magnetic field is trapped in the conducting plasma. Its subsequent evolution then can be followed as in ref. [8.

Having stated all these issues, we will take a simple path. We will assume, for simplicity, that strong magnetic field exists inside the plasma. The strength of magnetic field will be estimated close to its peak value, and will be assumed to survive in the plasma for the duration of evolution we carry out evolving according to the equations of relativistic magnetohydrodynamics (RMHD). We assume ideal RMHD with infinite conductivity, so magnetic field lines are frozen in the plasma. Due to our computer limitations (for our $3+1$ dimensional simulations), 
we are only able to consider small lattice, hence evolve for short times up to about $3 \mathrm{fm}$ (to avoid boundary effects). This being a rather short time, our assumption of ideal MHD may not be very inappropriate. Our focus in the paper is mainly on the qualitative aspects of results, and not on actual numbers. We are not claiming to give numbers which can be compared to the experimental data. Rather we demonstrate qualitative patterns of flow evolution, which one can look for in the experiments. Primary emphasis being on these being signals of the presence of strong magnetic field during early stages of plasma evolution.

The paper is organized in the following manner. In Sec.II, we briefly review the formalism we have adopted for the relativistic MHD simulation from ref. 14. Sec.III presents details of our numerical simulation. In Sec.IV we first discuss the issue of effect of magnetic field on elliptic flow (in view of conflicting conclusions in [3, 5, 6]). Sec.V presents results of the simulations which show that magnetic field can lead to qualitatively new effects. Sec.VI presents conclusions and discussions.

\section{THE FORMALISM}

We here provide a brief summary of the formalism we have followed for our relativistic magnetohydrodynamical (RMHD) simulations. For this we have followed ref. 14] and for the benefit of the reader we provide essential steps from that ref. in the following. We will be assuming zero baryon chemical potential situation so there will not be any baryon number conservation equation. Equations for ideal RMHD for the evolution of fluid and magnetic field are as follows.

Conservation of total energy momentum tensor (for QGP as well as the magnetic field) is given by

$$
\partial_{\alpha}\left[\left(\rho+p_{g}+|b|^{2}\right) u^{\alpha} u^{\beta}-b^{\alpha} b^{\beta}+\left(p_{g}+\frac{|b|^{2}}{2}\right) \eta^{\alpha \beta}\right]=0,
$$

where we have used perfect fluid form for the QGP energy-momentum tensor,

$$
T^{\mu \nu}=\left(\rho+p_{g}\right) u^{\mu} u^{\nu}+p_{g} \eta^{\mu \nu} .
$$

Maxwell's equations are

$$
\partial_{\alpha}\left(u^{\alpha} b^{\beta}-b^{\alpha} u^{\beta}\right)=0 .
$$

Here $\rho$ and $p_{g}$ are the energy density and pressure of QGP which we assume to be related by an ideal gas equation of state, $p_{g}=\frac{\rho}{3}$. $u^{\alpha}$ is the four-velocity with $u^{\alpha} u_{\alpha}=-1$. Four-vector $b^{\alpha}$ is related to the magnetic field $\vec{B}$ as,

$$
b^{\alpha}=\gamma\left[\vec{v} \cdot \vec{B}, \frac{\vec{B}}{\gamma^{2}}+\vec{v}(\vec{v} \cdot \vec{B})\right]
$$

$\gamma$ is the Lorentz factor for velocity $\vec{v}$ and we have following normalizations

$$
u^{\alpha} b_{\alpha}=0, \text { and }|b|^{2} \equiv b^{\alpha} b_{\alpha}=\frac{|\vec{B}|^{2}}{\gamma^{2}}+(\vec{v} \cdot \vec{B})^{2} \text {. }
$$

For numerical simulation, the above equations are cast in the following form

$$
\frac{\partial U}{\partial t}+\sum_{k} \frac{\partial F^{k}}{\partial x^{k}}=0
$$

This is the evolution equation for the vector $U$ where

$$
U=\left(m_{x}, m_{y}, m_{z}, B_{x}, B_{y}, B_{z}, E\right)
$$

where

$$
m_{k}=\left[\rho h \gamma^{2}+|\vec{B}|^{2}\right] v_{k}-(\vec{v} \cdot \vec{B}) B_{k}
$$

and

$$
E=\rho h \gamma^{2}-p_{g}+\frac{|\vec{B}|^{2}}{2}+\frac{v^{2}|\vec{B}|^{2}-(\vec{v} \cdot \vec{B})^{2}}{2} .
$$

$h$ is the specific enthalpy $\left(4 p_{g} / 3\right.$ with the ideal gas equation of state we are using for QGP) and $F^{k}$ are the fluxes in Eqn.(6) along directions $x^{k} \equiv(x, y, z)$ given as follows.

$$
F^{x}=\left(\begin{array}{c}
m_{x} v_{x}-B_{x} \frac{b_{x}}{\gamma}+p \\
m_{y} v_{x}-B_{x} \frac{b_{y}}{\gamma} \\
m_{z} v_{x}-B_{x} \frac{b_{z}}{\gamma} \\
0 \\
B_{y} v_{x}-B_{x} v_{y} \\
B_{z} v_{x}-B_{x} v_{z} \\
m_{x}
\end{array}\right)
$$

Similar expressions hold for $F^{y}$ and $F^{z}$ by appropriate replacement of indices. Here $p=p_{g}+\frac{|b|^{2}}{2}$ is the total pressure. Evolution is carried out using Eqn.(6) for the vector $U$ from which the independent variables $\left(p_{g}, \vec{v}, \vec{B}\right)$ have to be extracted. For this we define $W=\rho h \gamma^{2}$ and $S=\vec{m} \cdot \vec{B}$. With this we can write,

$$
\begin{gathered}
E=W-p_{g}+\left(1-\frac{1}{2 \gamma^{2}}\right)|\vec{B}|^{2}-\frac{S^{2}}{2 W^{2}}, \\
|m|^{2}=\left(W+|\vec{B}|^{2}\right)^{2}\left(1-\frac{1}{\gamma^{2}}\right)-\frac{S^{2}}{W^{2}}\left(2 W+|\vec{B}|^{2}\right) .
\end{gathered}
$$

This equation is used to express $\gamma$ as a function of $W$ and known variables $\vec{m}, \vec{B}$, and hence $S$ (from the knowledge of vector $U$ ). 


$$
\gamma=\left(1-\frac{S^{2}\left(2 W+|\vec{B}|^{2}\right)+|m|^{2} W^{2}}{\left(W+|\vec{B}|^{2}\right)^{2} W^{2}}\right)^{-1 / 2}
$$

With the ideal gas equation of state we have $p_{g}=\frac{W}{4 \gamma^{2}}$. Eqn.(9) then can be entirely written in terms of unknown quantity $W$ and other known quantities $\vec{B}, S$ and $E$ as follows

$$
f(W) \equiv W-p_{g}+\left(1-\frac{1}{2 \gamma^{2}}\right)|\vec{B}|^{2}-\frac{S^{2}}{2 W^{2}}-E=0 .
$$

We solve this equation using Newton-Raphson method to get $W$ using expressions for various derivatives as in ref. 14]. (Except for one derivative, we obtain $d p_{g} / d W$ using the equation $p_{g}=\frac{W}{4 \gamma^{2}}$ for our choice of equation of state. This expression differs from the expression in ref. 14.). From the value of $\mathrm{W}$ thus obtained, $\gamma$ can be calculated using Eqn.(13). With this, we get value of $p_{g}$. Equation for $m_{k}$ (Eqn.(8)) can then be used to obtain velocity components $v_{k}$. This completes the procedure of recovery of independent variables from time evolved vector $U$. For further details, we refer to ref.[14].

\section{DETAILS OF NUMERICAL SIMULATION}

We have developed a $3+1$ dimensional code and use lattice of size $200 \times 200 \times 200$ (and in some cases, for example for power spectrum for very strong magnetic field case, to get averages over several events we use smaller lattice $150 \times 150 \times 150$ ). For evolution we use leapfrog algorithm of 2 nd order accuracy. Due to small size of the lattice (due to computer limitations) we are able to evolve only for times up to $3 \mathrm{fm}$ to avoid boundary effects. In some cases we evolve for shorter times as we will mention for the respective cases.

Glauber like initial conditions are used for the initial energy density profile where a nucleus-nucleus collision is viewed as a sequence of independent binary nucleonnucleon collisions [15. The enhancement of $v_{2}$ is studied using smooth Glauber initial conditions in the X-Y plane. The parameters are tuned to an initial central temperature of $160-180 \mathrm{MeV}$ assuming energy density of ideal gas of quarks and gluons for the two flavor case with zero chemical potential. A smooth Woods-Saxon profile is used along $\mathrm{z}$-axis with extent equal to the extent of the colliding region along Y-axis. While studying the other effects like flux re-organisation, any possibility of vorticity generation, and the power spectrum, we use Glauber Monte Carlo initial conditions with parameters tuned to obtain the desired temperature range of $160-180 \mathrm{MeV}$. We distribute the energy density from the collision of participants along z-axis following a Gaussian distribution. As we mentioned above, ideal gas equation of state is used for QGP. We also add a constant background energy density of about $1 \%$ of the maximum initial energy density of the plasma, it gave better stability for the simulation, especially in the presence of fluctuations. (This energy density addition is not needed due to any instability of the program. Our algorithm of extracting the primitive variable does not work effectively when magnetic field energy density is much larger than the plasma density, as was noted in ref.6 also. Hence a non-zero energy density is used in the outer region. Such a small energy density should not affect any results strongly. Indeed, it is hard to argue that surroundings of the QGP region do not have some small energy density.) For some of the results, we have neglected fluctuations and have used Glauber optical initial conditions along the $x-y$ plane. This is done so that one can isolate the effects of magnetic field on the specific features of plasma evolution. Fluctuations lead to magnetic flux rearrangement which makes evolution highly complex, as we will demonstrate in the section on results. So, in the presence of fluctuations it becomes hard to associate specific patterns of flow with the magnetic field. Certainly, for experimental comparison one will need to combine all the effects together, and make special efforts to identify specific regimes of collision energy, nuclear size, centrality etc. to enhance the effects due to magnetic field. When we present results we specify where fluctuations are included and where not.

For the initial configuration of magnetic field we have used several methods. For most of the results we use magnetic field produced by two oppositely moving uniformly charged spheres (representing colliding nuclei), in vacuum, with appropriate Lorentz gamma factor [10, 12. This neglects modifications due to participants, but that is not expected to be very significant. This works fine with the range of magnetic fields expected in relativistic heavy-ion collisions, though we restrict our simulation to lower energy collisions about $\sqrt{s}=20 \mathrm{GeV}$. For most of the simulations below, the magnetic field profile is obtained in this manner. We typically give two sets of results, labeled by $B_{\text {time }}$ which is the time at which the magnetic field profile is calculated after the collision. We use $B_{\text {time }}=0.4 \mathrm{fm}$ and $0.6 \mathrm{fm}$. Smaller value gives larger value of the magnetic field, but may not be very realistic in view of finite conductivity of the plasma. If we use very large Lorentz gamma factor, then the magnetic field is sharply peaked at receding (Lorentz contracted) nuclei, and our $3+1$ dimensional simulation is not able to run for reasonable times, especially in the presence of fluctuations. For some cases just to show some interesting effects (e.g. systematic difference in the power of even-odd flow coefficients) we needed to use very high magnetic fields (of order $15 \mathrm{~m}_{\pi}^{2}$ ). Such large magnetic field are completely unrealistic here, and we use this value only to show how completely new effects may arise for very large magnetic field. The simulation with realistic magnetic field profile develops instabilities, primarily because in this case magnetic field energy density is much larger than the plasma density everywhere. 
Such difficulties have been noticed in other simulations as well 6] where it is mentioned that the numerical code was not able to handle configurations where the magnetic pressure is much larger than the thermal pressure, which typically is the case in regions outside the plasma region. To avoid these difficulties, for the large magnetic field case, we use a simpler profile for magnetic field where the profile in the $(\mathrm{x}-\mathrm{z})$ plane is chosen to be proportional to the energy density profile in the $(x-z)$ plane at $y=0$ obtained from the Glauber Monte Carlo like procedure as described above. $\mathrm{z}$ axis is the collision axis and the impact parameter is along the $\mathrm{x}$ axis, with resulting magnetic field pointing along the $y$ axis. The peak value of the magnetic field is chosen by hand. The magnetic field is then taken to be constant along the $\mathrm{y}$ axis, as consistent with Gauss' law. Clearly this magnetic field profile is not realistic along the y axis, but is only used to illustrate special effects of magnetic field on plasma evolution. The possibility remains that large magnetic fields may not be very unrealistic for example for deformed nucleus case.

We mention here that for low energy collisions with $\sqrt{s}=20 \mathrm{GeV}$ it is not appropriate to work with the simple zero chemical potential ideal relativistic gas equation of state which we have used. Also, at such low energies, chemical potential is sizeable and one cannot ignore baryon current. We use these approximations (simple equation of state and zero chemical potential) for simplicity, just as we have used ideal MHD equations for the evolution of the plasma. Our aim in this work is not to give definite numbers which can be compared with the experiments. Rather we look for basic physics for new effects. These qualitative patterns will not be expected to depend on the presence (or absence) of baryon current, or on the exact nature of the equation of state, though the numerical values will certainly depend on the factors. We thus expect that the qualitative patterns we find and the basic physics of new phenomena we discuss, will apply from low energy collisions (e.g. at FAIR and NICA) to high energy collisions at LHC.

As the simulation is carried out using ( $\mathrm{x}, \mathrm{y}, \mathrm{z})$ coordinates, with complete 3-dimensional profile for the initial energy density and magnetic field, we incorporate longitudinal expansion by assuming a maximum value of the velocity (of 0.7 ) at maximum $\mathrm{z}$ value for the Lorentz contracted energy density profile. (Note that this maximum velocity represents the velocity of the equilibrated plasma, and not that of the receding nuclei.) For intermediate distances, velocity is assumed to vary linearly as appropriate for Bjorken scaling. We use the lab time coordinate for time evolution. For the initial energy density profile, we first assume energy density profile as appropriate for an initial constant proper time hypersurface, evolving locally by longitudinal Bjorken scaling law, and then transform it to the constant lab time. This neglects nonlinear effects of inhomogeneities on evolution for a very short proper time period (the initial time for the beginning of plasma evolution), but should not be important for later time evolution. All our results are for the central rapidity region with unit rapidity window (suitably translated to $\Delta z$ ). This further makes our results reasonably reliable as the difference between the proper time and lab time are significant only for larger rapidities. Due to limitation of lattice size we have only considered small nucleus, copper in our case. Even for that, we have taken the radius to vary from about $3 \mathrm{fm}$ to $4.5 \mathrm{fm}$ (depending on consideration of fluctuations etc.). We again emphasize that the spirit of our work here is to demonstrate various important qualitative patterns in the flow in the presence of magnetic fields, rather than precise numbers.

\section{EFFECT OF MAGNETIC FIELD ON ELLIPTIC FLOW}

Before we present results of our simulations for different aspects of flow evolution, including the elliptic flow, we discuss previous results in the literature regarding effects of magnetic field on elliptic flow. In an earlier paper 3, some of us had argued that magnetic field can lead to enhancement of elliptic flow by up to about $30 \%$. We first briefly recall physical arguments for such an enhancement as discussed in ref. [3. Basic argument in [3] relied on the the effects of an external magnetic field on sound waves in QGP produced in RHICE. For relativistic magnetohydrodynamics, the waves which are relevant for the case of discussion of flow are the magnetosonic waves as they involve density perturbations. Phase velocities for these waves are given by 16

$\mathbf{v}_{p h}=v_{p h} \mathbf{n}=\mathbf{n}\left(\frac{1}{2}\left[\left(\rho_{0} h_{0} / \omega_{0}\right) c_{s}^{2}+v_{A}^{2}\right]\right)^{1 / 2}\left(1+\delta \cos ^{2} \theta \pm a\right)^{1 / 2}$.

Here + and - signs correspond to the fast and slow magnetosonic waves respectively, $v_{A}=B_{0} / \sqrt{\omega_{0}}$ is the Alfvén speed, and $\delta$ and $a$ are defined below. Mean local values of various quantities are denoted by subscript $O$ and $\theta$ is the angle between the magnetic field and $\mathbf{n}$.

$$
\begin{gathered}
a^{2}=\left(1+\delta \cos ^{2} \theta\right)^{2}-\sigma \cos ^{2} \theta \\
\delta=\frac{c_{s}^{2} v_{A}^{2}}{\left[\left(\rho_{0} h_{0} / \omega_{0}\right) c_{s}^{2}+v_{A}^{2}\right]}, \quad \sigma=\frac{4 c_{s}^{2} v_{A}^{2}}{\left[\left(\rho_{0} h_{0} / \omega_{0}\right) c_{s}^{2}+v_{A}^{2}\right]^{2}} .
\end{gathered}
$$

(Note, $\sigma$ is defined above, and should not be confused with the conductivity as used above.) For propagation of density perturbations, as relevant for the evolution of flow anisotropies, the relevant wave velocity is the group velocity for the magnetosonic waves,

$$
\mathbf{v}_{g r}=v_{p h}\left[\mathbf{n} \pm \mathbf{t} \frac{\left[\sigma \mp 2 \delta\left(a \pm\left(1+\delta \cos ^{2} \theta\right)\right)\right] \sin \theta \cos \theta}{2\left(1+\delta \cos ^{2} \theta \pm a\right) a}\right]
$$


Here $\mathbf{t}=\left[\left(\mathbf{B}_{\mathbf{0}} / B_{0}\right) \times \mathbf{n}\right] \times \mathbf{n}$, and again the upper and lower signs $( \pm$ or $\mp)$ correspond to the fast and the slow magnetosonic waves respectively. For a given magnetic field $\mathbf{B}_{\mathbf{0}}$, the direction of $\mathbf{n}$ can be varied to generate group velocities of these waves in different directions. Fig. 1 shows a typical situation of various vectors in Eq.(18) expected in RHICE. It is important to note that the direction of $\mathbf{v}_{g r}$ depends on the relative factors multiplying $\mathbf{n}$ and $\mathbf{t}$ in Eq.(18). This in turn depends on properties of the plasma like energy density. Thus due to the presence of spatial gradients in RHICE, especially due to initial state fluctuations, even along a fixed azimuthal direction, we expect the direction of $\mathbf{v}_{g r}$ to keep varying with the radial distance. This can lead to the development of very complex flow patterns. This raises a very interesting possibly of generation of vorticity in the plasma entirely from the effect of magnetic field. We are not able to fully explore this possibility as yet due to our limitation of relatively small time evolution of the plasma. (Vorticity will be expected to arise at later times when the flow pattern gets significantly twisted due to magnetic field effects.) Any such vorticity will have important implications, especially in view of chiral vortical effect.

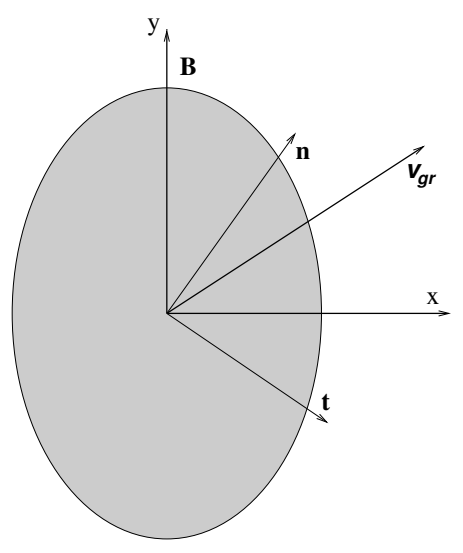

FIG. 1: A typical situation expected in relativistic heavy-ion collisions with the magnetic field pointing in the y direction. The direction of the group velocity $\mathbf{v}_{g r}$ is obtained from $\mathbf{n}$ and $\mathbf{t}$ via Eq.(18). (figure taken from ref. [3] ).

The effect of magnetic field on propagation of sound waves here comes from an effective magnetic pressure arising from the freezing of magnetic field lines in the plasma in the magnetohydrodynamics limit. The distortions of magnetic field lines in the presence of density perturbations cost energy leading to an extra contribution to pressure from the presence of magnetic field. This is what is responsible for increasing the effective sound speed as given above. The estimate of the effect of magnetic field on elliptic flow in ref. [3] was based on the fact that the flow coefficients are proportional to the sound velocity [17, which now becomes dependent on the directions of the magnetic field and that of the phase velocity.
This directly affects the flow pattern and hence elliptic flow.

We mention that these arguments are rather crude. Elliptic flow is a complex phenomenon and cannot be directly related to the anisotropy of the stiffness of equation of state and resulting sound velocity. Our intention here is to point out the underlying physics of the phenomena and why one may expect an increased elliptic flow from the presence of magnetic field. A more detailed analysis of the effects of magnetic field on elliptic flow was carried out by Tuchin in [5] with results in agreement with the estimates of 3. Later, in the section of results we will present results of our numerical simulation where again magnetic field is found to enhance elliptic flow. However, quite different results are reported in a recent numerical RMHD simulations where magnetic field was found to have no effect on elliptic flow [6. It is important to understand possible reasons for the discrepancies between these different works. For this purpose we have carried out simulations to study elliptic flow evolution with different values of impact parameters which lead to different types of magnetic field profiles. Our conclusion is that in the end the effects of magnetic field on flow pattern has many complex features. The picture used in $[3$ was indeed too simplistic where the magnetic field dependent sound speed was directly assumed to affect the elliptic flow. In fact quite opposite arguments could be given using Lenz's law from which one expects that induced magnetic fields will always oppose the change which causes magnetic flux changes. Basically this should imply that expansion along $\mathrm{x}$ axis should be suppressed as this leads to decrease in magnetic flux, while expansion along y axis should not be affected, thereby decreasing elliptic flow. The actual situation is much more complex. For example, Lenz's law argument does not distinguish between uniform expansion along $\mathrm{x}$ axis and the distortion of a localized plasma by transverse expansion. The latter leads to distortion of field lines, and not just decrease in magnetic flux, which has implications for extra pressure, and hence on sound waves. Some of the complexities have been discussed recently in refs. [18, 19], though exact time dependence used for magnetic field in ref.19 seems difficult to justify, (also for the Gaussian profile of the magnetic field in the x-y plane in ref.[18], one needs to ensure that Gauss' law is satisfied.)

As we will see later, net effect of magnetic field on elliptic flow depends very sensitively on the profile of magnetic field in relation to the profile of plasma energy density. When magnetic field is entirely localized within the plasma, we typically find enhancement of elliptic flow, in accordance with the physical arguments in 3]. However, when the magnetic field profile extends significantly beyond the plasma profile, plasma expansion seems to be hindered by the squeezing of external field lines, thereby suppressing elliptic flow. Presence of initial state fluctuations introduces extra complications due to flux rearrangements, as we will discuss below. It is possible that a combination of such effects may be responsible for dis- 
crepancies between these various results on the expected magnetic field dependence of elliptic flow.

\section{RESULTS}

We now present results of our simulations. As we mentioned, due to small lattice size, we are able to consider evolution for a maximum of only $3 \mathrm{fm}$ time to avoid boundary effects. We first present results for elliptic flow.

\section{A. Magnetic field dependence of elliptic flow}

We carry out simulations with different impact parameters and calculate elliptic flow with magnetic field and without magnetic field. The latter is calculated by repeating the same simulation, but with magnetic field switched off.

First we present results for the conventional momentum anisotropy defined as $\epsilon_{p}=\frac{T^{x x}-T^{y y}}{T^{x x}+T^{y y}}$. We calculate $\epsilon_{p}$ at different times with and without magnetic field. Fig. 2 shows these plots. As expected, $\epsilon_{p}$ increases gradually with time. However, we see that $\epsilon_{p}$ in the presence of magnetic field increases more rapidly, clearly showing enhancement of momentum anisotropy due to magnetic field (for this choice of parameters, in particular, with impact parameter of $4 \mathrm{fm}$ ).

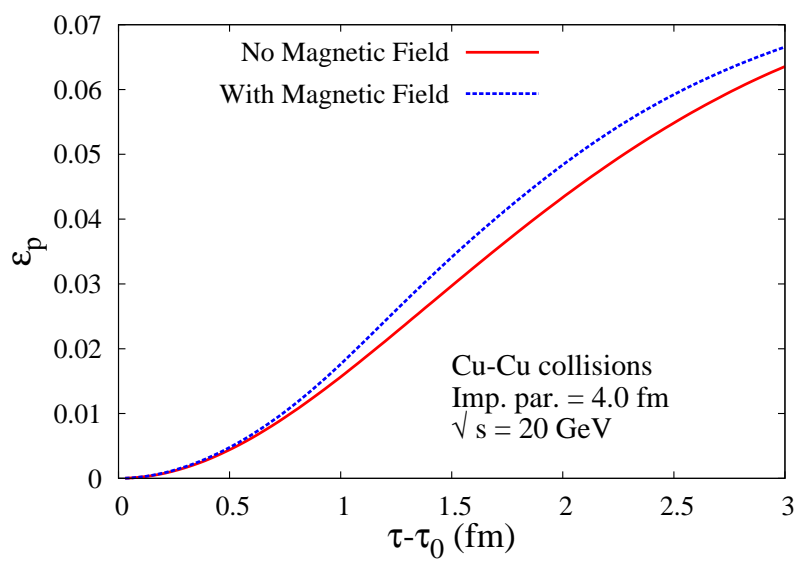

FIG. 2: Effect of magnetic field on build up of momentum anisotropy $\epsilon_{p}$, showing clear enhancement of $\epsilon_{p}$ with magnetic field, for this set of parameters, in particular for impact parameter of $4 \mathrm{fm}$.

Though this expression for momentum anisotropy represents the expected development of momentum anisotropy, we will not use this definition of momentum anisotropy. Instead, we will use Fourier expansion of the following normalized momentum anisotropy

$$
f(\phi)=\frac{\Delta p(\phi)}{\bar{p}}=\frac{p(\phi)-\bar{p}}{\bar{p}}
$$

$v_{2}$ is taken to be the 2nd Fourier coefficient in the Fourier series expansion of $f(\phi)$. Here $p(\phi)$ is the fluid momentum in a bin at azimuthal angle $\phi$ calculated from momentum components of the energy momentum tensor, i.e. from $T^{x 0}$ and $T^{y 0}$, integrating over the plasma volume in the central rapidity region of unit rapidity width. We believe that the expression for $v_{2}$ obtained from Eqn.(19) is more appropriate as it directly gives the momentum anisotropy as measured in the experiment, rather than expected momentum anisotropy $\epsilon$ defined in terms of $T^{x x}$ and $T^{y y}$. Interestingly, this definition of $v_{2}$ has a specific advantage over $\epsilon_{p}$. As Fig.2 shows, $\epsilon_{p}$ increases gradually, and becomes sizeable only after significant time (in Fig.2 at $t=3 \mathrm{fm}$ ). So, to study effects of magnetic field on momentum anisotropy in various conditions, it requires running simulation every time upto significant time. In contrast, the definition in Eqn.(19) gives a value of $v_{2}$ which has a large value right from the beginning (after first few time steps), it very slowly changes afterwards due to evolving shape of the plasma region. This may appear surprising, but there is a simple physical explanation for this behavior. Consider a definition of $v_{2}=\frac{T^{x 0}-T^{y 0}}{T^{x 0}+T^{y 0}}$. (It is simple to see that the arguments given for this $v_{2}$ apply to the definition of $v_{2}$ obtained from Fourier expansion of $f(\phi)$ in Eqn.(19).) One can see from the form of QGP energymomentum tensor (Eqn.(2)) that for small velocities (at initial times), this $v_{2}$ equals $\frac{v_{x}-v_{y}}{v_{x}+v_{y}}$. With initial fluid velocity directly proportional to the pressure gradient (as one can see from Euler's equation, see, e.g. [17]), we see that $v_{2}$ captures complete information about spatial anisotropy right from the beginning. It does not depend on the magnitude of the velocity, but only on the fractional difference in $v_{x}$ and $v_{y}$. As long as the fluid acceleration remains roughly constant, the value of $v_{2}$ above will remain roughly the same. essentially, the velocities (both $v_{x}$, and $v_{y}$, hence also fluid momenta) will simply increase with time. End result will be that time will not play much role for this definition of $v_{2}$. Same argument applies to $f(\phi)$ in Eqn.(19) and $v_{2}$ obtained from its Fourier expansion. That is the reason we find that $v_{2}$ assumes a large, roughly constant value right from the beginning stages, and starts changing later only with changes in the spatial anisotropy (and effects of fluctuations etc.). In contrast, the usual definition of momentum anisotropy $\epsilon_{p}$ is equal to (again, for small velocities at initial times) $\frac{v_{x}^{2}-v_{y}^{2}}{v_{x}^{2}+v_{y}^{2}+\frac{1}{2 \gamma^{2}}}$ with the equation of state $\rho=3 p_{g}$. This value increases from zero smoothly to finite value due to extra factor of $\frac{1}{2 \gamma^{2}}$ in the denominator as velocity magnitude increases in time. This is why we see $\epsilon_{p}$ in Fig.2 gradually increasing in time (for both cases, with and without magnetic field). For our case, $v_{2}$ in Eqn.(19) increases rapidly to a finite value simply because at the first stage itself the acceleration of the fluid (and hence the instantaneous velocity) completely originates from the anisotropy of pressure gradient arising from the spatial anisotropy. We find little change in 
the value of $v_{2}$ for significant initial time (of order 2-3 $\mathrm{fm}$ ), and after that it evolves primarily because of the changes in the spatial anisotropy, as expected. Thus, we believe it is much more appropriate to use the expression for $v_{2}$ obtained from Eqn.(19) rather than the usual one based on $T^{x x}$ and $T^{y y}$. This also helped us in collecting results for many runs with different impact parameters, with and without magnetic field, as the initial $v_{2}$ was itself found to be close to the time evolved value of $v_{2}$ up to several fm time.

Fig. 3 shows the effect of magnetic field on elliptic flow. Top figure in Fig. 3 shows the plot of $v_{2}(B) / v_{2}(0)$ vs. the impact parameter. We see clear enhancement in $v_{2}$ due to magnetic field which reaches a peak value at the impact parameter of about $3 \mathrm{fm}$, decreasing subsequently. Interestingly, for large impact parameter (near about 6.5 $\mathrm{fm}$ ) there is no effect of magnetic field on $v_{2}$ and for larger impact parameters, magnetic field actually leads to suppression of $v_{2}$, with suppression being strong for impact parameter of $8 \mathrm{fm}$. The bottom figure in Fig. 3 shows the behavior of $v_{2}$ for the cases of without magnetic field (solid, red curve) and with magnetic field (dashed and dotted curves) separately, clearly showing that for large impact parameters, magnetic field strongly suppresses the elliptic flow. This is despite the fact that the magnetic field is monotonically increasing function of the impact parameter almost for the entire range considered, as can be seen in Fig.4, with only slight decrease for the case $B_{\text {time }}=0.4 \mathrm{fm}$ (that cannot account for the decrease of $v_{2}(B)$ which is seen for both values of $B_{\text {time }}=0.4$ and $0.6 \mathrm{fm})$. We will discuss below the physical reasons for this behavior which will also explain the discrepancies in the results of refs. [3, 5] and ref. [6]. In all the figures, we typically give two curves labeled by $B_{\text {time }}$ which is the time at which the magnetic field profile is calculated after the collision. Smaller value of $B_{\text {time }}$ gives larger value of the magnetic field, but may not be very realistic in view of finite conductivity of the plasma.

We have studied the reason for this non-trivial behavior of magnetic field dependence of elliptic flow and it appears to originate from the differences in the profiles of magnetic field vs. the energy density profile. For smaller values of impact parameters, the magnetic field profile is reasonably confined while the plasma density profile extends for larger regions. This is the regime where arguments in [3, 5] seem to be valid and enhancement of $v_{2}$ is seen in the presence of magnetic field. This situation is shown in Fig.5 which shows the initial profile of the magnetic field as well as the initial energy density profile for impact parameter of $1 \mathrm{fm}$.

Quite opposite profiles are seen in Fig.6 which shows initial profiles for magnetic field and energy density for a large impact parameter of $7 \mathrm{fm}$. (For both Figs.5,6 we have used $B_{\text {time }}=0.4 \mathrm{fm}$.) Extension of significant strength of magnetic field profile beyond the plasma profile along $\mathrm{x}$ axis (semi-minor axis of the elliptical QGP shape) squeezes plasma expansion in $\mathrm{x}$-direction as magnetic field lines in the outer regions offer stiffness against
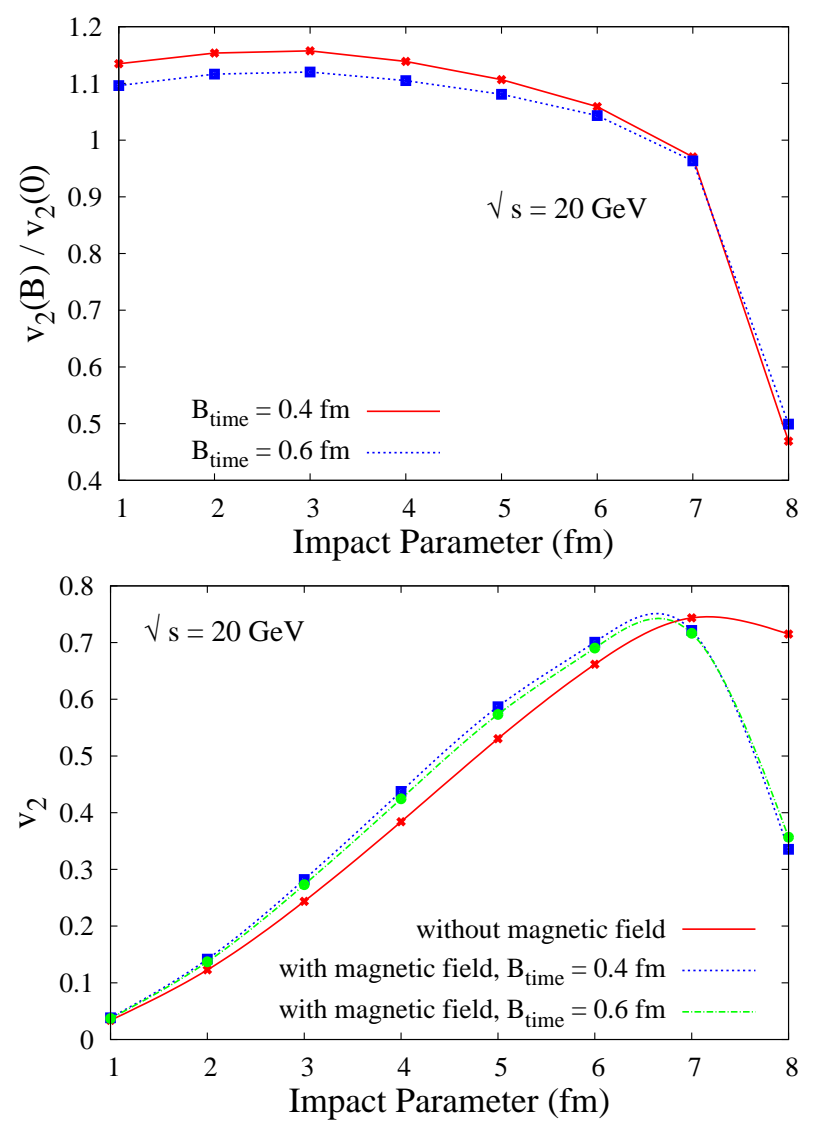

FIG. 3: Top figure shows the plot of $v_{2}(B) / v_{2}(0)$ vs the impact parameter. The ratio peaks at the impact parameter of about $3 \mathrm{fm}$, decreasing afterwards, and actually becomes less than 1 (meaning suppression of elliptic flow due to magnetic field) for large impact parameters. Bottom figure shows the plots of $v_{2}$ for the cases of without magnetic field (solid,red, curve) and with magnetic field (dashed and dotted curves) separately, clearly showing that for large impact parameters, magnetic field strongly suppresses the elliptic flow.

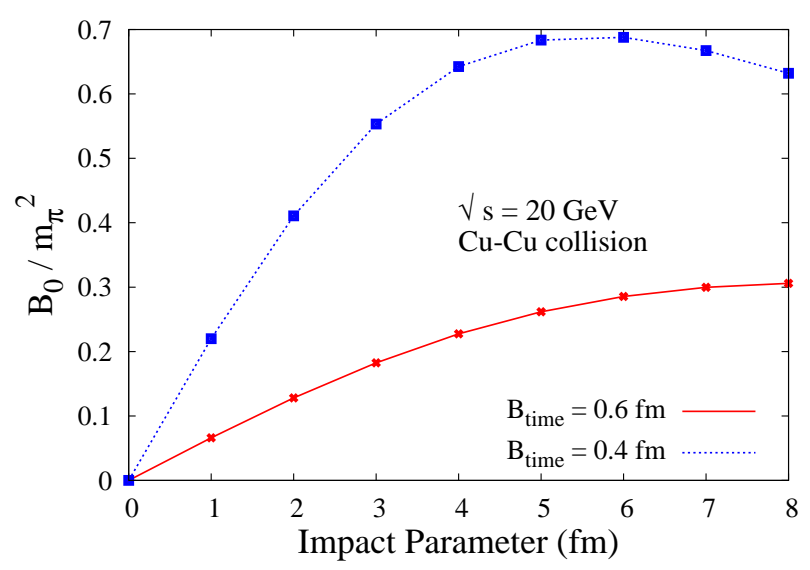

FIG. 4: Central value of magnetic field as a function of the impact parameter. Note that magnetic field almost monotonically increases with the impact parameter. 

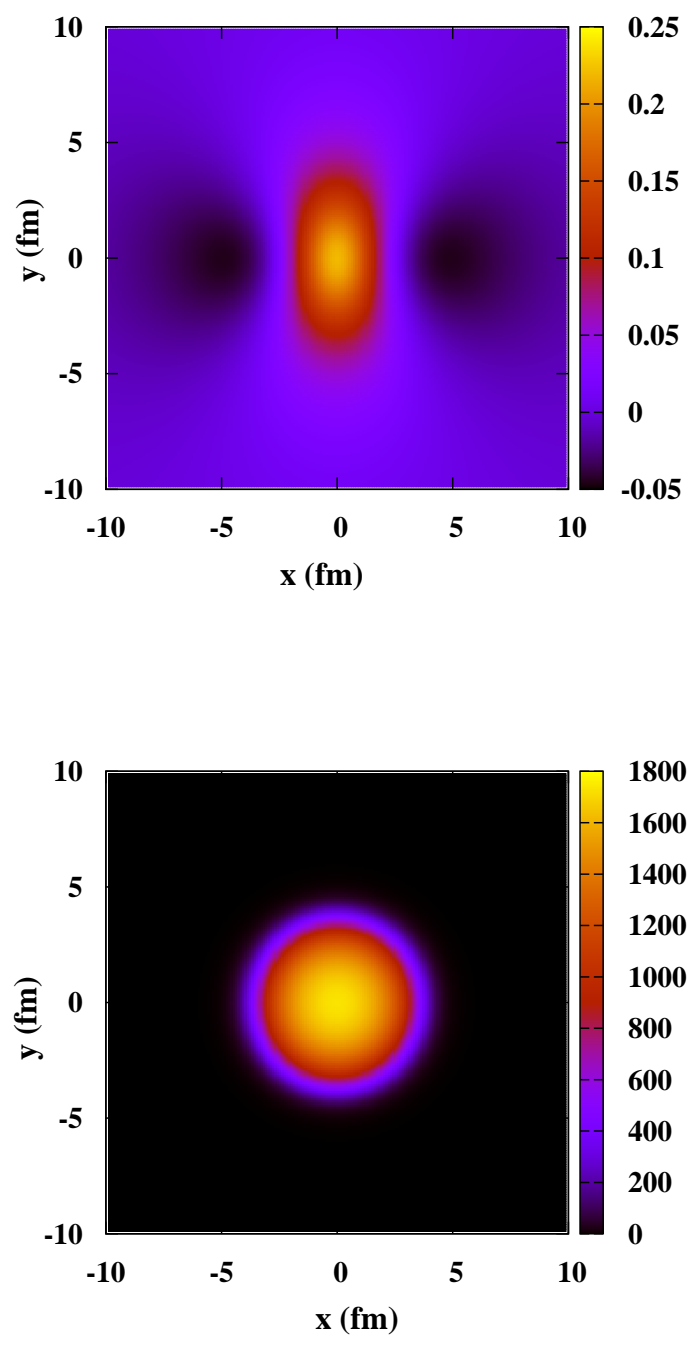

FIG. 5: Top figure shows the initial magnetic field profile for impact parameter of $1 \mathrm{fm}$. Bottom figure shows the initial plasma energy density profile for the same case. Note that for this small value of impact parameter, plasma extends well beyond the region along $\mathrm{x}$-axis where magnetic field is significant. Here and in Fig. 6 we show the y component of the magnetic field (in the units of $m_{\pi}^{2}$, the energy density in the units of $\left.\mathrm{MeV} / \mathrm{fm}^{3}\right)$.

distortion. This seems to be the cause of decrease in $v_{2}(B) / v_{2}(0)$ for larger impact parameters. This is especially demonstrated by the very strong decrease in $v_{2}(B)$ for impact parameter beyond $7 \mathrm{fm}$ in the bottom figure in Fig.3. (Note in this context, that simulations in [6], where no effect of magnetic field was found on the elliptic flow, were carried out for $\mathrm{Au}-\mathrm{Au}$ collisions with large impact parameter.).

Our conclusion of this investigation is that the effect
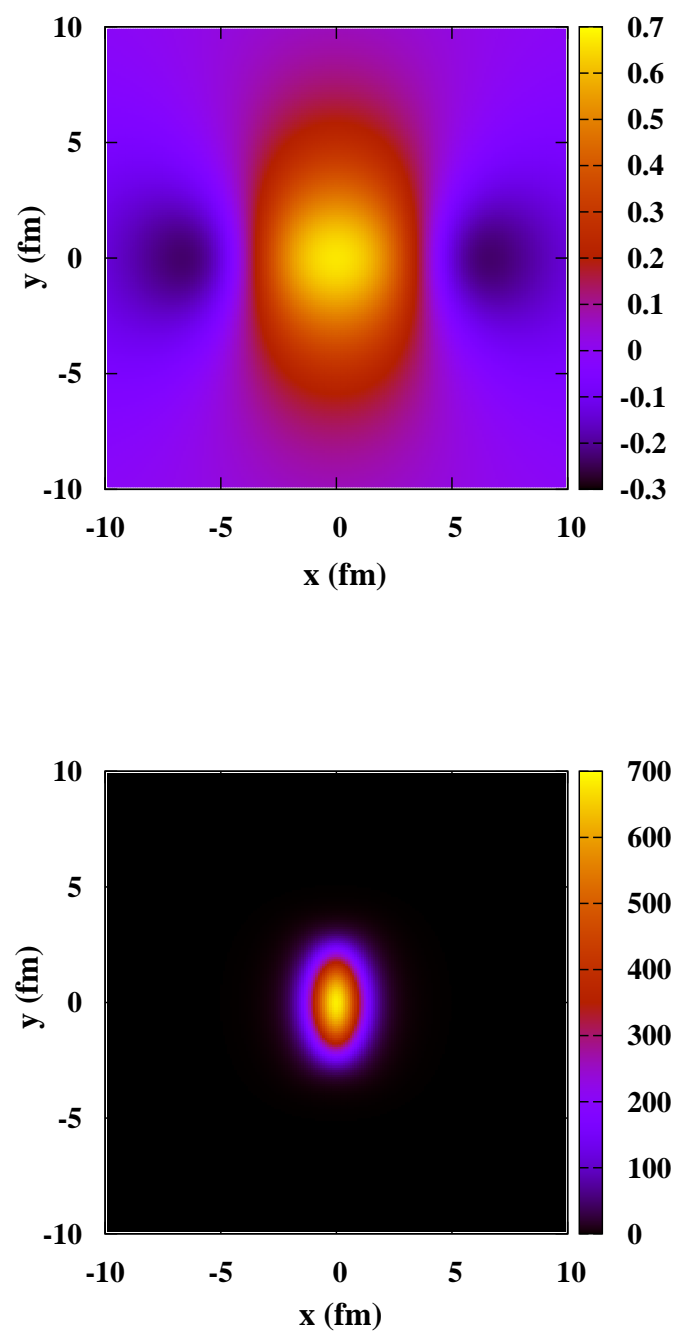

FIG. 6: Top figure shows the initial magnetic field profile for impact parameter of $7 \mathrm{fm}$. Bottom figure shows the initial plasma energy density profile for the same case. Note that for this large value of impact parameter, the two profiles show opposite behavior compared to Fig.5. Here we see that the magnetic field profile extends beyond the region along $\mathrm{x}$-axis compared to the plasma energy density profile.

of magnetic field on elliptic flow is quite complex. There are several physical effects at play here, from anisotropic sound speed due to magnetic field direction (which tends to increase elliptic flow), to Lenz's law which suppresses plasma expansion in the regime of external magnetic field (which tends to suppress elliptic flow). Net effect on the elliptic flow depends on which factors dominate. We are not attempting to provide a definitive answer to the discrepancies between different results for $v_{2}(B) / v_{2}(0)$ in the literature, but pointing out possible factors which 
may be responsible for this. Nonetheless, the strong suppression of elliptic flow in the presence of magnetic field for large impact parameters may provide a signal for the presence of strong magnetic field during early stages of plasma evolutions.

\section{B. Magnetic flux re-arrangement due to fluctuations}

One usually expects that magnetic field decreases as plasma evolves. It is indeed true at an average level. However we know that the plasma has strong initial state fluctuations in the energy density. As fluctuations evolve, the dynamics of magnetic flux lines (which are mostly frozen in the plasma) become very complex. It is clearly possible that in some region plasma expansion dilutes the magnetic flux, while due to energy density inhomogeneities, the neighboring region may get concentration of magnetic flux, thereby locally increasing the magnetic field. We find that indeed this happens. Fig.7 shows the plot of central magnetic field for two different cases. The thin curve (with stars) shows the case for Gaussian width of $0.3 \mathrm{fm}$ for the energy deposition in each binary collision in Glauber Monte Carlo, while the thick curve (with solid squares) represents the case of Gaussian width of $0.4 \mathrm{fm}$, thereby representing a much smoother background for the plasma. We see that for this smoother plasma case, the magnetic field roughly monotonically decreases with time (after a very little initial increase, again due to relatively small fluctuations) as expected. However, for the case of smaller Gaussian width, representing stronger fluctuations, the magnetic field initially increases significantly almost by about $10 \%$, and eventually decreases. This is only a sample case, and it is clear that for stronger fluctuations, one may expect even stronger temporary increase of the magnetic field during plasma evolution. This can have important consequences for effects like chiral magnetic effect and chiral vortical effect (with a possibility that complex flow pattern arising from magnetic field in the presence of fluctuations can in principle lead to generation of vortices). These effects strongly depend on the presence of topological charge density (for chiral magnetic effect) and vorticity (for chiral vortical effect). These quantities are reasonably localized, and if the magnetic field in these relevant regions tends to increase in time (for some time) it can lead to strong enhancement of these effects compared to the usual expectation based on decreasing magnetic field.

\section{Effects of magnetic field on the power spectrum of flow fluctuations}

We now consider the effects of magnetic field on the power spectrum of flow fluctuations. Power spectrum of flow fluctuations for a large number of flow coefficients can be a very valuable source for investigating early

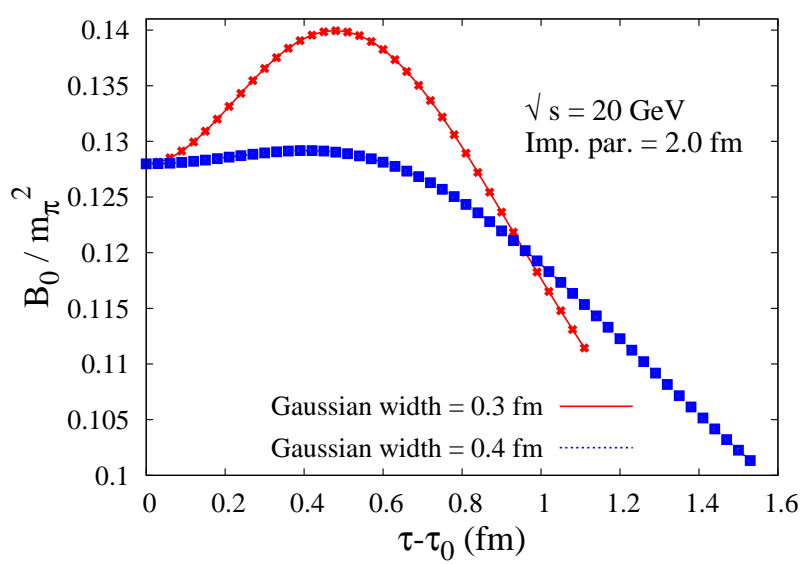

FIG. 7: Plot of central magnetic field in the presence of fluctuations and for relatively smoother plasma back ground. We see that for the smoother case, the magnetic field monotonically decreases as expected. However, for the case of stronger fluctuations, the magnetic field initially increases, and eventually decreases.

stages of plasma evolution [20]. The reason for departure from conventional focus on only first few even flow coefficients was the recognition that initial state fluctuations contribute to development of all flow coefficients (including the odd ones) even for a central collisions. Many subsequent investigations confirmed this expectation [21] and indeed now one routinely measures odd coefficients (e.g. the triangular flow coefficient $v_{3}$ ) and there have also been several investigations of power spectrum of flow coefficients upto a large value of $n$ of about 10-12. From the discussion above it is obvious that magnetic field will affect the power spectrum in non-trivial manner. Indeed, it was an earlier calculation of effects of primordial magnetic field on CMBR power spectrum 22 which prompted some of us to explore the possibility of magnetic field effects on the power spectrum of flow fluctuations in RHICE 3 .

We use same methods for calculating flow anisotropies as in our earlier work 20]. $v_{n}$ denotes the $n_{t h}$ Fourier coefficient of the resulting momentum anisotropy in $\delta p / p$. We do not calculate the average values of the flow coefficients $v_{n}$, instead we calculate root-mean square values of the flow coefficients $v_{n}^{r m s}$. Further, these calculations are performed in a lab fixed frame, without any reference to the event planes of different events. Average values of $v_{n}$ are zero due to random orientations of different events. As $v_{n}^{r m s}$ will have necessarily non-zero values, physically useful information will be contained in the non-trivial shape of the power spectrum (i.e. the plot of $v_{n}^{r m s}$ vs. $n$ ). We show below in Fig.8 the effects of magnetic field on the power spectrum calculated after time evolution of about $2 \mathrm{fm}$. These results are for realistic magnetic field for the collision energy considered here $(\sqrt{s}=20$ $\mathrm{GeV}$ ) for copper nuclei with central field strength of 0.1 $m_{\pi}^{2}$ and $0.4 m_{\pi}^{2}$ corresponding to $B_{\text {time }}=0.6$ and $0.4 \mathrm{fm}$ 
respectively (with initial state fluctuations). (For the results for the power spectrum calculations, we have taken initial longitudinal velocity of the plasma to be zero for the stability of the program in the presence of strong fluctuations.) As we can see, the effects of magnetic field are very tiny, though they are clearly present. As we will see below, the effects of magnetic field are not seen prominently here due to the effects of fluctuations being dominant for the power spectrum. Limited particle statistics may make it very difficult to observe such tiny effects.

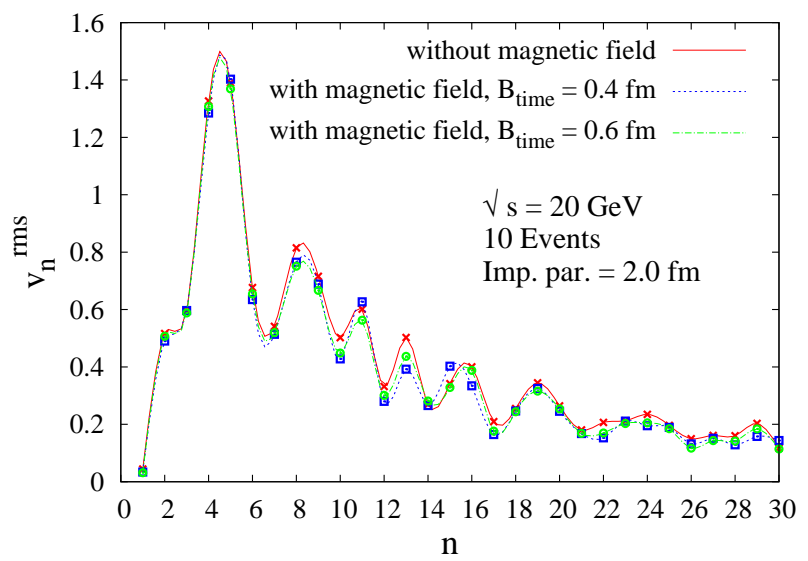

FIG. 8: Plot of $v_{n}^{r m s}$ with and without magnetic field. Even though magnetic field affects the power spectrum, its effects are very tiny here for the magnetic field considered here $(0.1$ and $\left.0.4 m_{\pi}^{2}\right)$.

As we mentioned in the Introduction, it is of great importance to find signals which can indicate the presence of strong magnetic field during the initial stages. Fig.8 shows possible effects of magnetic field, though the effects are very insignificant for these low magnetic fields (for much larger field appropriate for large values of $\sqrt{s}$, e.g. at LHC, these effects may become significant. We are not able to carry out simulations for such large values of $\sqrt{s}$ at present.) Further, the effects seen in Fig.8 do not show any qualitatively distinct pattern for the power spectrum. We show qualitatively different result below for very strong magnetic fields. We consider magnetic field strength to be $5 m_{\pi}^{2}$ and $15 m_{\pi}^{2}$. These values are completely unrealistic here (unless unexpected things happen, say for deformed nuclei), and we use these only to show how completely new effects can arise for very large magnetic field. As we mentioned in Sect.III, for large magnetic fields, requiring large Lorentz gamma factor, the realistic magnetic field profile (as used for Fig.8) causes problems with simulation. Thus for these cases (for Figs.9,10,11 below), we use a simpler profile for magnetic field where the profile in the $(\mathrm{x}-\mathrm{z})$ plane is chosen to be proportional to the energy density profile in the $(x-z)$ plane at $y=0$. The peak value of the magnetic field is chosen by hand. The magnetic field is then taken to be constant along the y axis, as consistent with the Gauss's law. Fig.9 and Fig.10 below show the power spectrum for magnetic field of strength $5 \mathrm{~m}_{\pi}^{2}$ and $15 \mathrm{~m}_{\pi}^{2}$ respectively. As these are runs for very strong magnetic field, simulation could be carried out only for relatively short time of 0.6 $\mathrm{fm}$. We see strong pattern of different powers in even and odd $v_{n}^{r m s}$ coefficients. This is expected from the reflection symmetry about the magnetic field direction if initial state fluctuations are not dominant. Note that for $5 m_{\pi}^{2}$ case, even-odd pattern is seen for only first few flow coefficients as fluctuation effects wash out the effect for larger $v_{n}$ for the event average over 10 events. For $15 m_{\pi}^{2}$ case the magnetic field is very strong and fluctuation effects are not able to wash out the even-odd pattern arising from the magnetic field. This is a qualitatively distinct result and can give unambiguous signal for the presence of strong magnetic field during early stages.

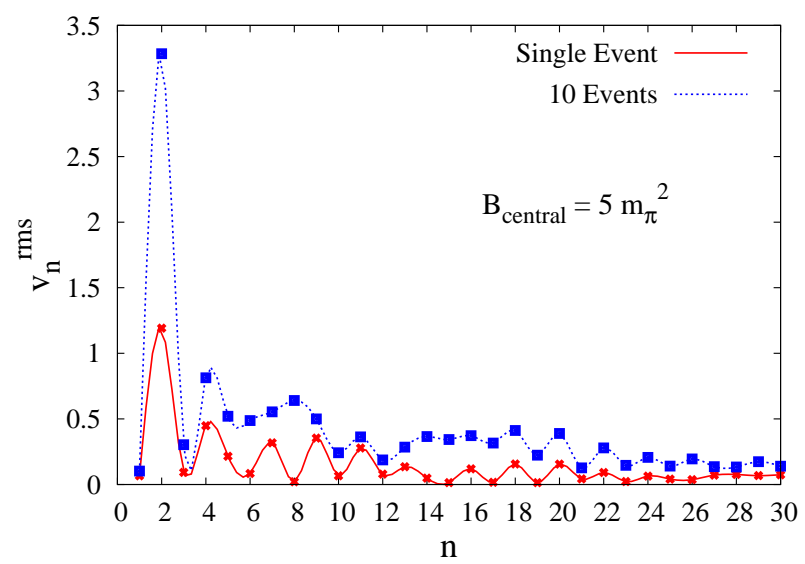

FIG. 9: Plot of $v_{n}^{r m s}$ for magnetic field with strength $5 m_{\pi}^{2}$. Even-odd power difference is seen in first few flow coefficients as fluctuations wash out the effect for large $v_{n} \mathrm{~s}$.

The reason one needs very strong magnetic field is that although magnetic field tends to develop clear pattern of even-odd power difference, there are strong effects of initial state fluctuations on the power spectrum. The final power spectrum is a combined effect of the two patterns. Strong magnetic field is needed to dominate over the effects of fluctuations in Fig.9,10. To illustrate this, we show in Fig.11 flow fluctuations for a smooth isotropic plasma region (without any fluctuations) in the presence of magnetic field. We now take a more reasonable value of magnetic field strength equal to $m_{\pi}^{2}$. Due to smaller magnetic field and smooth plasma profile, the evolution could be run up to $3 \mathrm{fm}$ time (after which boundary effects could not be neglected). We see that strong evenodd power difference is present in the power spectrum.

We mention that such even-odd power difference can arise due to presence of vortices also during early plasma evolution, as demonstrated in our earlier work [23]. Thus, we may conclude that even-odd difference in the power spectrum indicates either strong magnetic field or presence of vortices in the initial plasma. (We know that to some extent the effect of magnetic field in a plasma is sim- 


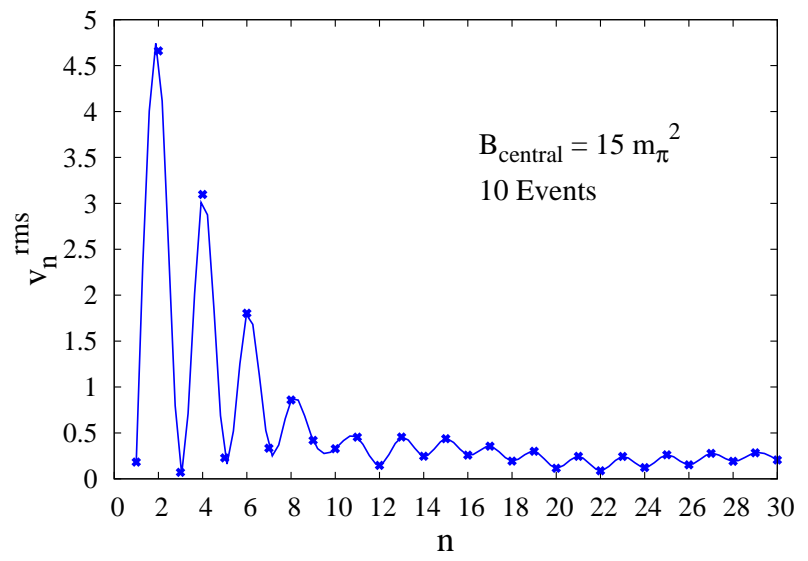

FIG. 10: Plot of $v_{n}^{r m s}$ for very strong magnetic field with strength $15 m_{\pi}^{2}$. Strong difference in the power of even and odd values of $v_{n}^{r m s}$ are seen. Though such large magnetic field are completely unexpected here, such effects may provide unambiguous signal for the presence of any unexpected strong initial magnetic field.

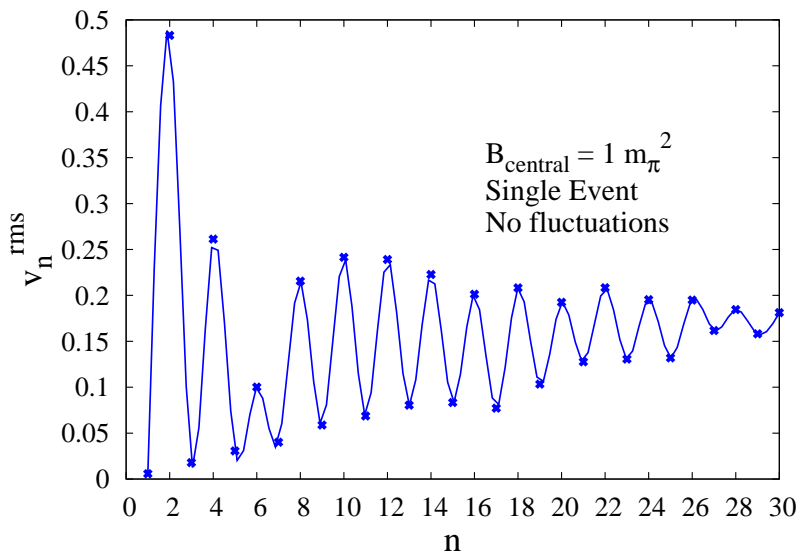

FIG. 11: Plot of $v_{n}^{r m s}$ for magnetic field with strength $m_{\pi}^{2}$. Here we consider isotropic region with smooth plasma profile without any fluctuations. Strong difference in the power of even and odd values of $v_{n}^{r m s}$ are present arising from the effect of magnetic field.

ilar to the presence of vortices as the Lorentz force due to magnetic field has similar form as the Coriolis force in the presence of vortices.) This result also has interesting implications for the CMBR power spectrum. It is known that low $l$ modes of CMBR power spectrum also show possible difference in even-odd modes [24]. It is possible that this feature may be indicative of the presence of a magnetic field, or presence of some vorticity, during the very early stages of the inflation.

\section{Anomalous elliptic flow for deformed nucleus}

Collision of deformed nuclei opens up entirely new range of possibilities for heavy-ion collisions. This is especially true when considering possible magnetic field configurations for a given shape of plasma. For non-central collisions of spherical nuclei, one is constrained to consider the magnetic field pointing along the semi-major axis of the elliptical QGP region. (Though due to fluctuations, deviations from this will happen but roughly the picture remains the same.) For deformed nuclei, entirely new possibilities can arise. As an example, Fig.12 shows ellipsoidal nuclei, with longer axes of both along the y axis, with impact parameter also along the y axis. As one can see from Fig.12, different impact parameters can lead to following anomalous magnetic field configurations (in the sense that they cannot arise for spherical nuclei).

a) QGP region being elliptical in shape but the magnetic field pointing along the semi-minor axis, $\mathrm{x}$-axis in this case as seen in Fig.12a.

b) QGP region being roughly spherical, but still strong magnetic field is present due to strong components coming from spectators, as seen in Fig.12b.

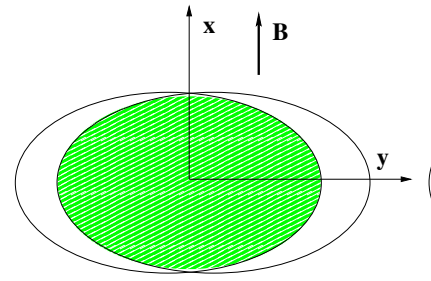

(a)

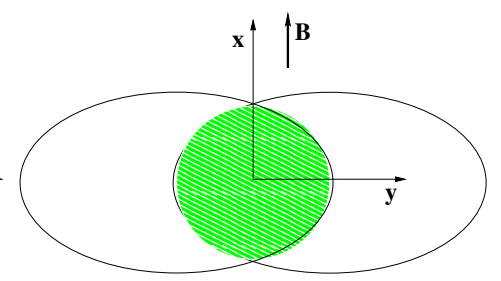

(b)
FIG. 12: (a) shows the situation of the case when the QGP region is elliptical in shape but the magnetic field points along the semi-minor axis. (b) shows the case when the QGP region is roughly spherical, but still strong magnetic field is present due to strong components coming from spectators.

With the physics of effects of magnetic field as described above, one can immediately guess what to expect in both these cases. For (a) we expect suppression of elliptic flow as the magnetic field induced anisotropy leads to larger momentum flowing in the direction of semimajor axis of the elliptical QGP shape, even though the usual fluid pressure gradient develops larger flow along the semi-minor axis. This leads to strong suppression of elliptic flow due to this anomalous magnetic field. (For very strong magnetic field the net $v_{2}$ may even be completely dominated by the magnetic field, leading to negative elliptic flow.) For (b) one would have expected no elliptic flow for the smooth plasma profile considered here, (non-zero $v_{2}$ may only arise from any fluctuations), as the QGP is roughly isotropic. However, the presence of strong magnetic field introduces anisotropic pressure, leading to development of non-zero $v_{2}$, even though QGP region is spherical. Figs.13,14 confirm these expectations. 
Again, the anomalous elliptic flow in these situations may provide a signal for initial stage magnetic field.

Note that here we are not simulating collision of deformed nuclei. We use the plasma profile for Fig.12a and Fig.12b by using collisions of spherical nuclei (copper) with non-zero and zero impact parameter respectively. But for the magnetic field we calculate the magnetic field as in Sects.5A and 5B, rotate it along the $\mathrm{x}$ axis, and use that for the evolution of the above plasma profiles. This, in some sense, models different situations of collisions of deformed nuclei as in Fig.12a,b. A full simulation for deformed nuclei is presently under investigation and will be presented in a future work.

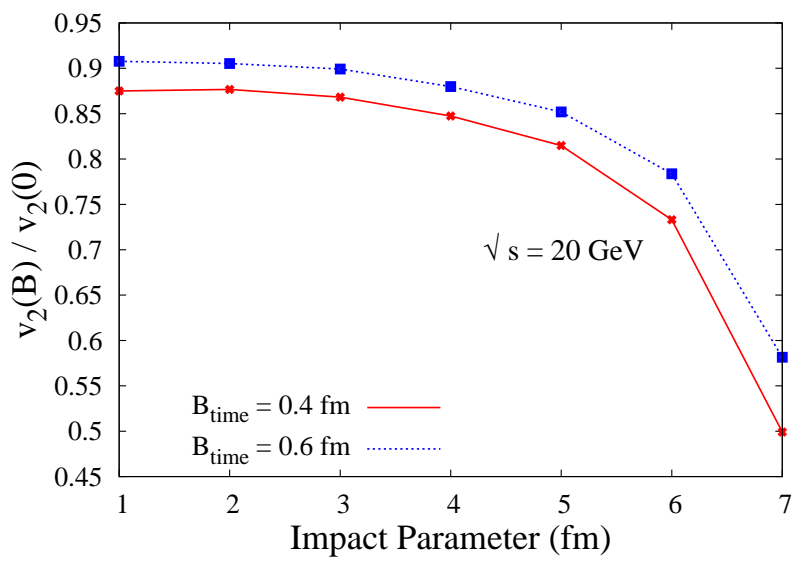

FIG. 13: $v_{2}$ for the case when the QGP region is elliptical in shape but the magnetic field points along the semi-minor axis, $\mathrm{x}$-axis in this case. Strong suppression of elliptic flow arises with larger momentum flowing in the direction of semi-major axis of the elliptical QGP shape due to magnetic field induced anisotropy.

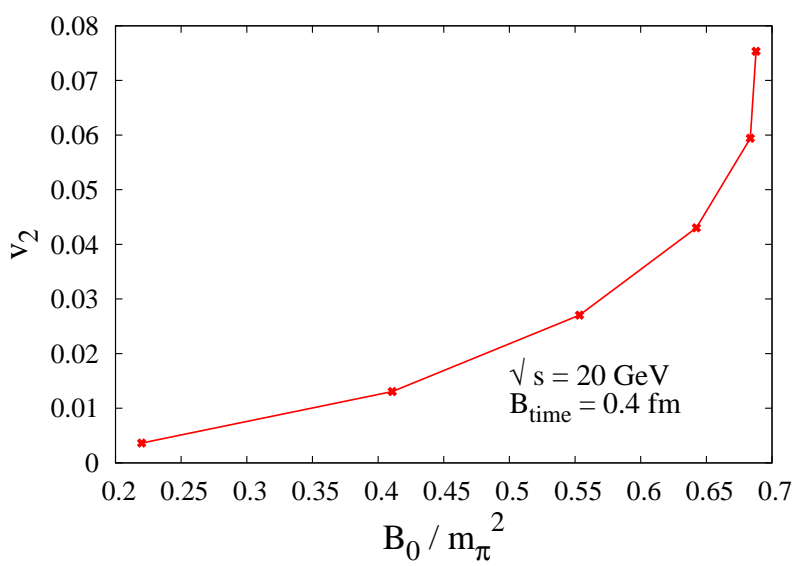

FIG. 14: $v_{2}$ for the case when the QGP region is roughly isotropic in shape but still non-zero magnetic field is present leading to non-zero $v_{2}$, monotonically increasing with the strength of magnetic field, even though no elliptic flow is expected in this case.

\section{E. Quadrupole magnetic field from deformed nucleus}

A very interesting possibility arises when considering collision of deformed nuclei. Consider again ellipsoidal nuclei with long axes in the transverse plane (as in the above), but now in crossed configuration. Fig.15 shows this crossed configuration for Uranium nucleus with the semi-minor and semi-major axes being about $6.7 \mathrm{fm}$ and $8.7 \mathrm{fm}$ respectively. Magnetic field is calculated at time of $0.4 \mathrm{fm}$ after the collision for $\sqrt{s}=20 \mathrm{GeV}$. It is clear that while the resulting QGP region is roughly isotropic (possibly with strong $v_{4}$ component), spectators will generate quadrupolar magnetic field as one can see from Fig.16 showing the magnetic field lines for this crossed configuration of colliding nuclei. (Magnetic field here has been calculated by extending the calculation of Sec.V A,B for the case of deformed nucleus, Uranium in this case. We calculate magnetic field from uniformly charged ellipsoidal nuclei [10, 25, oppositely moving, with appropriate Lorentz transformations.) This raises very important possibilities. Quadrupolar field will itself contribute to $v_{4}$, thereby affecting final value of $v_{4}$ of the plasma. Further, quadrupolar field will tend to focus plasma motion along the longitudinal direction, thereby affecting Bjorken longitudinal expansion itself. For charged plasma with finite conductivity one may expect charge separation in the transverse direction as a function of rapidity, while a focusing effect should be seen along the beam axis for the plasma. This should lead to suppression of transverse flow at non-zero rapidity. Further, if focusing is strong, it may lead to hot extended regions along the longitudinal axis. This requires a detailed investigation of plasma dynamics with such a crossed configuration collision of deformed nuclei properly represented in Glauber Monte Carlo. This is under study and will be presented in a future work.

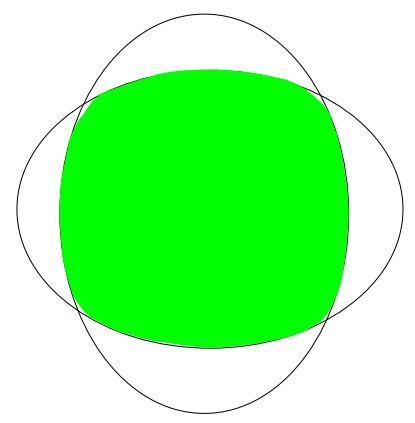

FIG. 15: Crossed configuration of collision of deformed nuclei. Note that the overlap region will be reasonably isotropic, with possibly strong $v_{4}$ component. Importantly now there are four spectator parts whose motion should lead to quadrupolar magnetic field configuration. 


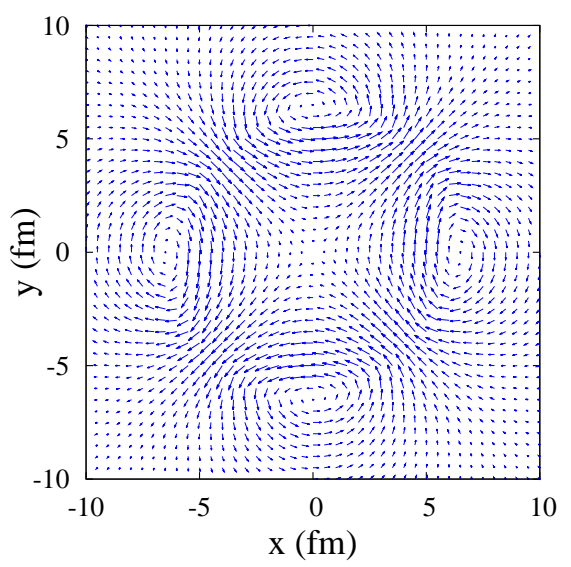

FIG. 16: Magnetic field configuration arising from collision of crossed deformed nuclei (Uranium) as in Fig.15. Quadrupolar nature of the field is clear.

\section{CONCLUSIONS}

We have demonstrated qualitatively new effects on the flow pattern of QGP in the presence of initial magnetic field. As we emphasized, due to various limitations of our simulation, we are not in a position to provide numbers which can be compared to experimental data. Our intention is to show possibilities of new physical phenomena which one can try to look for in experiments. These qualitative patterns may be able to provide clear signal for the presence of strong magnetic field during early stages of the evolution, though actual value of magnetic field etc. will depend on more reliable numerical estimates of the numbers. Among our results one of the results shows that due to flux re-arrangement arising from evolving fluctuations, there may be local regions where magnetic field increases for some time (before it starts decreasing finally). If topological charges or vortices are also present in that region, it can lead to enhancement of chiral magnetic/vortical effects. We see very complex patterns of twisting flow developing due to magnetic field effects in the presence of fluctuations. For strong fluctuations and strong magnetic field, it seems entirely possible that localized vorticity may get generated at later times which we are not able to study due to limitations of our simulation. Our result on enhancement of elliptic flow in the presence of magnetic field confirms earlier expecta- tion in refs. 3, 5. At the same time our simulation also points out that the effects of magnetic field on elliptic flow are much more complex than envisaged in simple arguments of ref. 3 . In fact in some situations one finds decrease in the elliptic flow. This may resolve the discrepancy between the results of ref. [3, 5] and ref. [6] (see, also refs. 18, 19]). The strong suppression of elliptic flow for large impact parameters can provide a signal for initial stage strong magnetic field. (For this it is needed to have observations extended for very large impact parameters, to distinguish from the suppression from usual hydrodynamics resulting from decreased plasma pressure at large impact parameters.) We show non-trivial effects of magnetic field on the power spectrum of flow fluctuations. The strongest form of this effect being in the form of even-odd power difference in the flow power spectrum for strong magnetic fields which can be a very clean signal for strong magnetic field, or vortices [23, in RHICE. (At the same time, it can have important implications for the low $l$ modes for CMBR power spectrum.) Our results for deformed nuclei provide possibilities of anomalous elliptic flow, which can be used to detect the magnetic field in such collisions. It points to a very interesting possibility of generating a quadrupolar magnetic field configuration which can have focusing effect on plasma in the longitudinal direction (along with a possibility of charge separation in the transverse direction.) These possibilities are under investigation at present and will be presented in a future work.

\section{Acknowledgments}

We are very grateful to Partha Bagchi, Srikumar Sengupta, Nirupam Dutta, Oindrila Ganguly, Ranjita Mohapatra, and Ananta P. Mishra for useful comments and discussions. Some of the results here were presented by AMS in the conferences QCD Chirality Workshop 2015 held at Physics Dept. UCLA (USA), in Jan. 2015, and in March 2017, and at the conference Hadronic Matter under Extreme Conditions held at JINR (Russia) in Nov. 2016, by AD in the conference Quark Matter 2017 at Chicago, Feb. 2017, and by SSD at the conference XQCD-2017 at Pisa, Italy, June 2017. We thank the participants in these meetings for very useful comments and suggestions.
[1] D. Kharzeev, R.D. Pisarski, and M.H.G. Tytgat, Phys. Rev. Lett. 81, 512 (1998); S.A. Voloshin, Phys. Rev. C 70, 057901 (2004).

[2] D.E. Kharzeev, L.D. McLerran, and H.J. Warringa, Nucl. Phys. A 803227 (2008).

[3] R. K. Mohapatra, P. S. Saumia, and A. M. Srivastava, Mod. Phys. Lett. A 26, 2477 (2011).
[4] P. Romatschke and U. Romatschke, Phys. Rev. Lett. 99, 172301 (2007).

[5] K. Tuchin, J.Phys. G39, 025010 (2012).

[6] G. Inghirami et al. arXiv: 1609.03042; Eur. Phys. J. C 76, 659 (2016).

[7] M. Asakawa, A. Majumder, and B. Muller, Phys. Rev. C 81, 064912 (2010). 
[8] K. Tuchin, Phys.Rev. C 83, 017901 (2011); Phys. Rev. C 82, 034904 (2010).

[9] L. Landau and E. Lifshitz, Electrodynamics of Continuous Media (Pergamon Press, N.Y., USA, 1984), Sect. 58.

[10] J.D. Jackson, Classical Electrodynamics, 3rd Edition (John Wiley \& Sons, Inc., USA, 1999).

[11] H.-T. Ding et al. arXiv:1012.4963, Phys. Rev. D 83, 034504 (2011).

[12] K. Tuchin, Phys. Rev. C 88, 024911 (2013).

[13] P.V. Buividovich, et al., Phys. Rev. Lett. 105, 132001 (2010).

[14] A. Mignone and G. Bodo, Mon. Not. R. Astron. Soc. 368, 1040 (2006).

[15] M.L. Miller, K. Reygers, S.J. Sanders, and P. Steinberg, Ann. Rev. Nucl. Part. Sci. 57, 205 (2007); P.F. Kolb, J. Sollfrank, and U. W. Heinz, Phys.Rev. C 62, 054909 (2000).

[16] J.P. (Hans) Goedbloed, R. Keppens, and S. Poedts, Advanced Magnetohydrodynamics (Cambridge University Press, UK, 2010).

[17] J.-Y. Ollitrault, Eur. J. Phys. 29, 275 (2008); R. S. Bhalerao, J. P. Blaizot, N. Borghini, and J.-Y. Ollitrault,
Phys. Lett. B 627, 49 (2005).

[18] L.G. Pang, G. Endrodi, and H. Petersen, Phys. Rev. C 93, 044919 (2016).

[19] S. Pu and D.L. Yang, Phys. Rev. D 93, 054042 (2016).

[20] A. P. Mishra, R. K. Mohapatra, P. S. Saumia, and A. M. Srivastava, Phys. Rev. C 77, 064902 (2008); Phys. Rev. C 81, 034903 (2010).

[21] P. Sorensen, J.Phys. G 37 (2010) 094011; B. Alver and G. Roland, Phys. Rev. C 81 (2010) 054905; A. Mocsy and P. Sorensen, Nucl. Phys. A 855 (2011) 241; J.I. Kapusta, Nucl. Phys. A 862-863 (2011) 47; J.Y. Ollitrault, J. Phys. Conf. Ser. 312 (2011) 012002; P. Sorensen, arXiv:0808.0503

[22] J. Adams, U.H. Danielsson, D. Grasso, and H. Rubinstein, Phys. Lett. B 388, 253 (1996).

[23] A. Das, S.S. Dave, S. De, and A.M. Srivastava, arXiv: 1607.00480, to appear in Mod. Phys. Lett. A.

[24] P.K. Aluri and P. Jain, Mon. Not. Roy. Soc. 419, 3378 (2012).

[25] O.D. Kellogg, Foundations of Potential Theory, (Springer-Verlag, 1967). 